Pacific

Journal of

Mathematics

\title{
THE SUBREPRESENTATION THEOREM FOR AUTOMORPHIC REPRESENTATIONS
}

MARCELA HANZER 


\title{
THE SUBREPRESENTATION THEOREM FOR AUTOMORPHIC REPRESENTATIONS
}

\author{
MARCEla HANZER
}

\begin{abstract}
We prove that every irreducible subrepresentation in the space of automorphic forms on $G(A)$, where $G$ is a connected reductive group defined over a number field $k$, and $A$ is the related ring of adeles, is a subrepresentation of the representation induced from a cuspidal automorphic representation of a Levi subgroup.
\end{abstract}

\section{Introduction}

In this note we prove the global (automorphic) version (over a number field $k$ ) of Casselman's subrepresentation theorem. We explain it in more detail: in the local theory (i.e., considering admissible representations of reductive groups over local fields) there is Harish-Chandra's subquotient theorem [1954], and then there is also Casselman's subrepresentation theorem [1980; 1995]; both of them state that every irreducible representation (in the appropriate category) of this given reductive group is a subquotient or (in the case of Casselman's theorem) a subrepresentation of a representation induced from a "simpler" one (of an appropriate subgroup). The global analog of the Harish-Chandra subquotient theorem would be Langlands' theorem which describes a general automorphic representation as a subquotient of a representation induced from a cuspidal representation of a Levi subgroup.

We prove the following global version of Casselman's subrepresentation theorem.

Theorem. Let $G$ be a connected reductive group defined over $k$. Let $(\Pi, V)$ be an $\left(\left(\mathfrak{g}_{\infty}, K_{\infty}\right) \times G\left(\mathbb{A}_{f}\right)\right)$-irreducible subspace of automorphic forms in $A(G(k) \backslash G(\mathbb{A}))$. Then, there exists a parabolic subgroup $P=M U$ of $G$, an irreducible automorphic cuspidal representation $\pi_{0}$ of $M$ (thus appearing in the space of cuspidal automorphic forms on $M$ ) such that, as abstract global representations, we have

$$
\Pi \hookrightarrow \operatorname{ind}_{P(\mathrm{~A})}^{G(\mathbb{A})} \pi_{0},
$$

where we consider the normalized parabolic induction (so we extend $\pi_{0}$ trivially on $U(\mathbb{A}))$ and we take $\mathbf{K}$-finite vectors.

Keywords: automorphic representation, subrepresentation, cuspidal representation. 
We explain all the notation in the Preliminaries section.

We are sure that the experts in the field are aware of the above claim, but we were not able to find the reference for this statement, which is somewhat more precise than the aforementioned Langlands' result in his Corvallis lecture [Borel and Jacquet 1979]. The proof is a pretty straightforward application of the Langlands proof in his Corvallis lecture, with the decomposition results (on the spaces of automorphic forms) obtained (along with much stronger results) in [Mœglin and Waldspurger 1995]. We hope that this result will be very helpful for explicit calculations with automorphic forms, since it is explicitly applicable to the discrete (and $\mathbf{K}$-finite) part of automorphic $L^{2}$ situation.

\section{Preliminaries}

Let $k$ be a number field, and $\mathbb{A}$ its ring of adeles. Let $G$ be a connected reductive group defined over $k$, and $G_{\infty}=\prod_{v} G\left(k_{v}\right)$, where the product is over archimedean places of $k$. We further denote $G\left(\mathbb{A}_{f}\right)=\prod_{v<\infty}^{\prime} G\left(k_{v}\right)$. Let $u$ be the enveloping algebra of the complexified Lie algebra $\mathfrak{g}$ of $G_{\infty}$ (and $\mathfrak{g}_{\infty}$ is the Lie algebra of $G_{\infty}$ ). We follow the notation of the first chapter of [Mœglin and Waldspurger 1995]. We denote by $\mathfrak{z}$ the center of $\mathcal{U}$ and by $K_{v}$ a maximal compact subgroup of $G\left(k_{v}\right)$, where $K_{v}=G\left(O_{k_{v}}\right)$ for almost all $v<\infty$. Here $O_{k_{v}}$ is the ring of integers in $k_{v}$. We set $K_{\infty}=\prod_{v \mid \infty} K_{v}$ and $\mathbf{K}=\prod_{v} K_{v}$. We fix a minimal parabolic subgroup $P_{0}$ of $G$ defined over $k$, and consequently, standard parabolic subgroups (defined over $k$ ) with respect to $P_{0}$. We denote by $S$ a maximal $k$-split torus of $G$, chosen inside $P_{0}$ and by $\Delta$ the set of simple $k$-roots of $G$ with respect to $S$ (and $P_{0}$ ). We know that each standard $k$-parabolic subgroup corresponds to a subset $\theta$ of $\Delta$. We denote this by putting $P=P_{\theta}$. We denote the modular function on $P$ by $\delta_{P}$. For a standard Levi $k$-subgroup $M$ of $G$, we denote by $\mathfrak{z}^{M}$ the analogue of $\mathfrak{z}$ for group $M$. We denote by $Z_{M}$ the center of $M$.

We use the following definition of an automorphic form: Let $P=M U$ be a standard $k$-parabolic subgroup of $G$ and $\phi: U(\mathbb{A}) M(k) \backslash G(\mathbb{A}) \rightarrow \mathbb{C}$ a function. We say that $\phi$ is automorphic if it satisfies the following conditions:

(1) $\phi$ has moderate growth (see [Mœglin and Waldspurger 1995, I.2.3]).

(2) $\phi$ is smooth (see [Mœglin and Waldspurger 1995, I.2.5]).

(3) $\phi$ is $\mathbf{K}$-finite.

(4) $\phi$ is $\mathfrak{z}$-finite.

Note that the space $A(U(\mathbb{A}) M(k) \backslash G(\mathbb{A}))$ of all automorphic forms as above can be related to the usual situation with the automorphic forms on $M(k) \backslash M(\mathbb{A})$ by attaching to each $k \in \mathbf{K}$ and $\phi$ as above a function $\phi_{k}: M(k) \backslash M(\mathbb{A}) \rightarrow \mathbb{C}$ defined by $\phi_{k}(m)=\delta_{P}^{-1 / 2}(m) \phi(m k)$ by noting that $\phi$ is automorphic if and only if it is smooth, 
K-finite, and for all $k \in \mathbf{K}, \phi_{k}$ is an automorphic form on $M(k) \backslash M(\mathbb{A})$. We denote by $A_{0}(U(\mathbb{A}) M(k) \backslash G(\mathbb{A}))$ the cuspidal part of the space $A(U(\mathbb{A}) M(k) \backslash G(\mathbb{A}))$; i.e., the space of all automorphic forms $\phi$ from $A(U(\mathbb{A}) M(k) \backslash G(\mathbb{A}))$ with the property that for every standard $k$-parabolic subgroup $P^{\prime}=M^{\prime} U^{\prime}$ such that $P_{0} \subset P^{\prime} \varsubsetneqq P$ we have $\phi_{P^{\prime}}=0$ (the constant term along $P^{\prime}$, defined by $\left.\phi_{P^{\prime}}(g)=\int_{U^{\prime}(k) \backslash U^{\prime}(\mathbb{A})} \phi(u g) \mathrm{d} u\right)$.

The space $A(U(\mathbb{A}) M(k) \backslash G(\mathbb{A}))$ is a module for the action of $\left(\mathfrak{g}_{\infty}, K_{\infty}\right) \times G\left(\mathbb{A}_{f}\right)$, i.e., for the global idempotent Hecke algebra $\mathscr{H}=\mathscr{H}_{\infty} \otimes \mathscr{H}_{f}$, where $\mathscr{H}_{\infty}$ is related to $\mathcal{U}$ and finite measures on $K_{\infty}$, and $\mathscr{H}_{f}=\otimes_{v<\infty}^{\prime} \mathscr{H}_{v}$, where $\mathcal{H}_{v}, v<\infty$ is the Hecke algebra of compactly supported, locally constant functions on $G\left(k_{v}\right)$ (see [Borel and Jacquet 1979 , Section 4]). Note that $A_{0}(U(\mathbb{A}) M(k) \backslash G(\mathbb{A}))$ is a submodule of $A(U(\mathbb{A}) M(k) \backslash G(\mathbb{A}))$ with this action. Note that the constant term (with respect to some standard $k$-parabolic subgroup $P=M U$ ) is an intertwining operator between $A(G(k) \backslash G(\mathbb{A}))$ and $A(U(\mathbb{A}) M(k) \backslash G(\mathbb{A}))$ [Mœglin and Waldspurger 1995, I.2.6].

Let $\xi$ be a character of $Z_{M}(k) \backslash Z_{M}(\mathbb{A})$, and let $\pi$ be an irreducible submodule of $A(M(k) \backslash M(\mathbb{A}))$, for a standard $k$-Levi subgroup $M$ of $G$. We denote by $A(M(k) \backslash M(\mathbb{A}))_{\pi}$ the isotypic submodule attached to $\pi$ (in the theorem below we deal with cuspidal $\pi$, so the relevant subquotients are indeed subspaces). We set

$$
\begin{aligned}
& A(U(\mathbb{A}) M(k) \backslash G(\mathbb{A}))_{\xi}=\{\phi \in A(U(\mathbb{A}) M(k) \backslash G(\mathbb{A})): \\
&\left.\phi(z g)=\delta_{P}^{1 / 2}(z) \xi(z) \phi(g) \text { for all } z \in Z_{M}(\mathbb{A}), g \in G(\mathbb{A})\right\}, \\
& A(U(\mathbb{A}) M(k) \backslash G(\mathbb{A}))_{\pi}=\{\phi \in A(U(\mathbb{A}) M(k) \backslash G(\mathbb{A})): \\
&\left.\phi_{k} \in A(M(k) \backslash M(\mathbb{A}))_{\pi} \text { for all } k \in \mathbf{K}\right\} .
\end{aligned}
$$

Analogously, we define by $A_{0}(U(\mathbb{A}) M(k) \backslash G(\mathbb{A}))_{\xi}$ and $A_{0}(U(\mathbb{A}) M(k) \backslash G(\mathbb{A}))_{\pi}$ the cuspidal parts of the above spaces (i.e., the parts realized in the space of cuspidal automorphic forms).

Proposition 2.1. Let $\xi$ be a character of $Z_{M}(k) \backslash Z_{M}(\mathbb{A})$ and let $\Pi_{0}(M)_{\xi}$ denote the set of isomorphism classes of irreducible representations of $M(\mathbb{A})$ occurring as submodules in $A_{0}(M(k) \backslash M(\mathbb{A}))_{\xi}$. We have the decomposition

$$
A_{0}(U(\mathbb{A}) M(k) \backslash G(\mathbb{A}))_{\xi}=\bigoplus_{\pi \in \Pi_{0}(M)_{\xi}} A_{0}(U(\mathbb{A}) M(k) \backslash G(\mathbb{A}))_{\pi}
$$

Proof. This is explained in [Mœglin and Waldspurger 1995, p. 44].

Remark. By the proof of Lemma I.3.2 of [Mœglin and Waldspurger 1995], $\mathfrak{z}^{M}$ acts on $A(U(\mathbb{A}) M(k) \backslash G(\mathbb{A}))$ by left translations; every automorphic form there is $\mathfrak{z}^{M}$-finite; analogously every element of that space is $Z_{M}(\mathbb{A})$-finite, again here $Z_{M}(\mathbb{A})$ acts by left translations (because we examine $\mathbf{K}$-finite automorphic forms). Also, it is easy to see that $A_{0}(U(\mathbb{A}) M(k) \backslash G(\mathbb{A}))$ is $Z_{M}(\mathbb{A})$-invariant subspace with this $Z_{M}(\mathbb{A})$-action. 


\section{The theorem}

In this section we prove the main theorem stated in Section 1. The proof follows directly from the next theorem, so our embedding from the main theorem is realized through the calculation of the constant term.

Theorem 3.1. Let $(\Pi, V)$ be an $\left(\left(\mathfrak{g}_{\infty}, K_{\infty}\right) \times G\left(\mathbb{A}_{f}\right)\right.$-) irreducible subspace of automorphic forms inside $A(G(k) \backslash G(\mathbb{A}))$ such that some constant term of a function from $V$ does not vanish along a $k$-parabolic subgroup $P_{\theta}$ of $G$; assume that $\theta$ is minimal (set of simple roots) with this property. Then, there exists an irreducible automorphic representation $\pi_{0}$ of $M_{\theta}(\mathbb{A})$ (appearing in $A_{0}\left(M_{\theta}(k) \backslash M_{\theta}(\mathbb{A})\right.$ ) such that the space of constant terms of $V$ along $P_{\theta}$, denoted by $V_{0}$, belongs (up to a left translation by an element from $\left.Z_{M_{\theta}}(\mathbb{A})\right)$ to the space $A_{0}\left(U_{\theta}(\mathbb{A}) M_{\theta}(k) \backslash G(\mathbb{A})\right)_{\pi_{0}}$ of cuspidal automorphic forms.

Proof. Let $f \in V$. By definition, the constant term $f_{P_{\theta}}(g)=\int_{U_{\theta}(k) \backslash U_{\theta}(\mathbb{A})} f(u g) \mathrm{d} u$ belongs to $A\left(U_{\theta}(\mathbb{A}) M_{\theta}(k) \backslash G(\mathbb{A})\right)$, more precisely, to the cuspidal part of this space (because of the minimality of $\theta$; see [Mœglin and Waldspurger 1995, I.2.6, I.2.18]). By the remark above the Theorem, $Z_{M_{\theta}}(\mathbb{A})$ acts on $A_{0}\left(U_{\theta}(\mathbb{A}) M_{\theta}(k) \backslash G(\mathbb{A})\right)$ by left translations, and every function from this space is $Z_{M_{\theta}}(\mathbb{A})$-finite. For every $z \in Z_{M_{\theta}}(\mathbb{A})$, let $V_{0}^{z}=l(z) V_{0}$ (the action by left translations). We know that taking the constant term is intertwining operator, so $V_{0}$ (and $V_{0}^{z}$ ) is (as an abstract $\left(\mathfrak{g}_{\infty}, K_{\infty}\right) \times G\left(\mathbb{A}_{f}\right)$ - representation) irreducible and isomorphic to $V$. Let $W=$ $\sum_{z \in Z_{M_{\theta}}(\mathbb{A})} V_{0}^{z}$.

We prove that there exists $F \in W, F \neq 0$ such that $\operatorname{dim}_{\mathbb{C}} \operatorname{span}_{\mathbb{C}}\{l(z) F: z \in$ $\left.Z_{M_{\theta}}(\mathbb{A})\right\}=1$. Firstly, let $F \neq 0$ be an element from $W$ such that the dimension of the space $Y:=\operatorname{span}_{\mathbb{C}}\left\{l(z) F: z \in Z_{M_{\theta}}(\mathbb{A})\right\}$ is minimal. We claim that this dimension is one. Indeed, let us assume that this dimension (of $Y$ ) is greater than one. If, for every $a \in Z_{M_{\theta}}(\mathbb{A})$ acting on $Y$, the whole space $Y$ is an eigenspace for certain eigenvalue, it would mean that $l(a)$, for every $a$, acts as a scalar operator on $Y$, and then every one-dimensional subspace, (also the one spanned by $F$ ) would be $Z_{M_{\theta}}(\mathbb{A})$-invariant; a contradiction (this would mean that $Y$ is one-dimensional). So, there exists $a \in Z_{M_{\theta}}(\mathbb{A})$ with a nonzero eigenspace strictly smaller than $Y$, attached to an eigenvalue $\alpha \neq 0$. This means that $Y_{1}:=(l(a)-\alpha) Y$ is a proper subspace of $Y$. Let $F_{1}:=(l(a)-\alpha) F \in Y_{1} . F_{1}$ is obviously nonzero; otherwise $l(b) F$ would be an eigenvector of $l(a)$ for eigenvalue $\alpha$ for every $b \in Z_{M_{\theta}}(\mathbb{A})$, so that the whole $Y$ is an eigenspace for $\alpha$; a contradiction. Now, we easily see that the span of the set $\left\{l(b) F_{1}: b \in Z_{M_{\theta}}(\mathbb{A})\right\}$ is inside $Y_{1}$, which leads to contradiction with our choice of $F$.

So, we conclude that there exists a character $\xi$ of $Z_{M}(k) \backslash Z_{M}(\mathbb{A})$ such that

$$
l(z) F(g)=\delta_{P_{\theta}}^{1 / 2}(z) \xi(z) F(g) \quad \text { for all } g \in G(\mathbb{A}), z \in Z_{M_{\theta}}(\mathbb{A}) .
$$


Now, let $W_{0}$ denote the $\left(\mathfrak{g}_{\infty}, K_{\infty}\right) \times G\left(\mathbb{A}_{f}\right)$-subspace of $W$ generated by $F$. For every vector from this space, (1) holds. Now, since $W=\sum_{a \in Z_{M_{\theta}}(\mathbb{A})} V_{0}^{a}$, where $V_{0}^{a}$ are irreducible subspaces, $W$ is also a direct sum of irreducible subspaces (for example, [Lang 2002, Chapter XVII]), and every $\left(\mathfrak{g}_{\infty}, K_{\infty}\right) \times G\left(\mathbb{A}_{f}\right)$-submodule of $W$ is a direct summand. From this directly follows that $W_{0}$ has an irreducible submodule; indeed if $W=\bigoplus_{z \in I} V_{0}^{z}$, for some $I \subset Z_{M_{\theta}}(\mathbb{A})$, then some projection attached to this decomposition $p_{z}: W \rightarrow V_{0}^{z}$ is nonzero on $W_{0}$. Now Ker $p_{z} \cap W_{0}$ has a direct (invariant) complement $W_{1}$ in $W$, and it is easy to see that $W_{1} \cap W_{0}$ is an irreducible submodule of $W_{0}$. This means that we have found an irreducible subspace of $W$ (so necessarily isomorphic to $V$ i.e., to $V_{0}$ ) where the relation (1) holds. This realization of $V$ inside $A_{0}\left(U_{\theta}(\mathbb{A}) M_{\theta}(k) \backslash G(\mathbb{A})\right)_{\xi}$ is thus obtained through taking of (maybe translated) constant term along $P_{\theta}$. From Proposition 2.1 we have

$$
A_{0}\left(U_{\theta}(\mathbb{A}) M_{\theta}(k) \backslash G(\mathbb{A})\right)_{\xi}=\bigoplus_{\pi \in \Pi_{0}\left(M_{\theta}\right)_{\xi}} A_{0}\left(U_{\theta}(\mathbb{A}) M_{\theta}(k) \backslash G(\mathbb{A})\right)_{\pi},
$$

and, combining our embedding with an appropriate projection, we have obtained an embedding

$$
\Pi \hookrightarrow A_{0}\left(U_{\theta}(\mathbb{A}) M_{\theta}(k) \backslash G(\mathbb{A})\right)_{\pi_{0}},
$$

for some automorphic (cuspidal) representation $\pi_{0}$ of $M_{\theta}(\mathbb{A})$.

Note that the space $A_{0}\left(M_{\theta}(k) \backslash M_{\theta}(\mathbb{A})\right)_{\pi_{0}}$ is semisimple (Gelfand and PiatetskiShapiro; see [Borel and Jacquet 1979, Section 4]); so there exists an irreducible subspace $V_{0}^{\prime}$ of automorphic forms in $A_{0}\left(M_{\theta}(k) \backslash M_{\theta}(\mathbb{A})\right)_{\pi_{0}}$ (thus isomorphic to $\pi_{0}$ ) such that there is an embedding

$$
\Pi \hookrightarrow A_{0}\left(U_{\theta}(\mathbb{A}) M_{\theta}(k) \backslash G(\mathbb{A})\right)_{V_{0}^{\prime}}
$$

(the space on the right-hand side has an obvious meaning). We note that, as a $\left(\mathfrak{g}_{\infty}, K_{\infty}\right) \times G\left(\mathbb{A}_{f}\right)$-module, the latter space is isomorphic to the global representation ind $P_{\theta}^{G(\mathbb{A})} \pi_{0}$ (where we use normalized induction and $\mathbf{K}$-finite vectors in this space) [Kim 2004, Section 4.5]. This isomorphism can also be given explicitly by $\phi \mapsto \phi^{\prime}$, where $\phi^{\prime}(g)=\phi_{g}$ and $\phi_{g}(m)=\delta_{P_{\theta}}(m)^{-1 / 2} \phi(m g)$. This is easily checked to be $G(\mathbb{A})$-isomorphism on the space of the smooth (not necessarily K-finite automorphic forms), but then taking $\mathbf{K}$-finite vectors from both spaces, we get the claim (see the second and third lectures in [Cogdell 2004]). This, in turn, proves our main theorem from Section 1.

\section{Acknowledgements}

We want to thank Neven Grbac for the helpful discussions about automorphic representations, and to Goran Muić and Marko Tadić for their interest in our work. 
Also, we would like to thank the anonymous referee for suggestions, which improved the style of the presentation and clarified some mathematical imprecisions.

\section{References}

[Borel and Jacquet 1979] A. Borel and H. Jacquet, "Automorphic forms and automorphic representations", pp. 189-207 in Automorphic forms, representations and L-functions, 1 (Corvallis, OR, 1977), edited by A. Borel and W. Casselman, Proc. Sympos. Pure Math. 33, Amer. Math. Soc., Providence, RI, 1979. MR 81m:10055 Zbl 0414.22020

[Casselman 1980] W. Casselman, "Jacquet modules for real reductive groups", pp. 557-563 in Proceedings of the International Congress of Mathematicians (Helsinki, 1978), vol. 2, edited by O. Lehto, Acad. Sci. Fennica, Helsinki, 1980. MR 83h:22025 Zbl 0425.22019

[Casselman 1995] W. Casselman, "Introduction to the theory of admissible representations of p-adic reductive groups", preprint, 1995, http://www.math.ubc.ca/ cass/research/pdf/p-adic-book.pdf.

[Cogdell 2004] J. W. Cogdell, "Lectures on $L$-functions, converse theorems, and functoriality for GLn", pp. 1-96 in Lectures on automorphic L-functions, edited by J. W. Cogdell et al., Fields Inst. Monogr. 20, Amer. Math. Soc., Providence, RI, 2004. MR 2071506 Zbl 1066.11021

[Harish-Chandra 1954] Harish-Chandra, "Representations of semisimple Lie groups, II", Trans. Amer. Math. Soc. 76 (1954), 26-65. MR 15,398a Zbl 0055.34002

[Kim 2004] H. H. Kim, "Automorphic L-functions", pp. 97-201 in Lectures on automorphic Lfunctions, edited by J. W. Cogdell et al., Fields Inst. Monogr. 20, Amer. Math. Soc., Providence, RI, 2004. MR 2071507 Zbl 1066.11021

[Lang 2002] S. Lang, Algebra, 3rd ed., Graduate Texts in Mathematics 211, Springer, New York, 2002. MR 2003e:00003 Zbl 0984.00001

[Mœglin and Waldspurger 1995] C. Mœglin and J.-L. Waldspurger, Spectral decomposition and Eisenstein series: a paraphrase of scripture, Cambridge Tracts in Mathematics 113, Cambridge University Press, Cambridge, 1995. MR 97d:11083 Zbl 0846.11032

Received October 8, 2011. Revised July 23, 2012.

MARCELA HANZER

DEPARTMENT OF MATHEMATICS

UNIVERSITY OF ZAGREB

BIJENIČKA CESTA 30

10000 ZAGREB

CROATIA

hanmar@math.hr 


\title{
PACIFIC JOURNAL OF MATHEMATICS
}

\author{
msp.org/pjm
}

Founded in 1951 by E. F. Beckenbach (1906-1982) and F. Wolf (1904-1989)

\section{EDITORS}

V. S. Varadarajan (Managing Editor)

Department of Mathematics

University of California

Los Angeles, CA 90095-1555

pacific@math.ucla.edu

Paul Balmer

Department of Mathematics

University of California

Los Angeles, CA 90095-1555

balmer@math.ucla.edu

Daryl Cooper

Department of Mathematics

University of California

Santa Barbara, CA 93106-3080 cooper@math.ucsb.edu

Jiang-Hua $\mathrm{Lu}$

Department of Mathematics

The University of Hong Kong

Pokfulam Rd., Hong Kong jhlu@maths.hku.hk
Don Blasius

Department of Mathematics University of California

Los Angeles, CA 90095-1555

blasius@math.ucla.edu

Robert Finn

Department of Mathematics Stanford University

Stanford, CA 94305-2125

finn@math.stanford.edu

Sorin Popa

Department of Mathematics

University of California

Los Angeles, CA 90095-1555

popa@math.ucla.edu

Paul Yang

Department of Mathematics

Princeton University

Princeton NJ 08544-1000

yang@math.princeton.edu

\section{PRODUCTION}

Silvio Levy, Scientific Editor, production@msp.org

\section{SUPPORTING INSTITUTIONS}

ACADEMIA SINICA, TAIPEI

CALIFORNIA INST. OF TECHNOLOGY

INST. DE MATEMÁTICA PURA E APLICADA

KEIO UNIVERSITY

MATH. SCIENCES RESEARCH INSTITUTE

NEW MEXICO STATE UNIV.

OREGON STATE UNIV.

\author{
STANFORD UNIVERSITY \\ UNIV. OF BRITISH COLUMBIA \\ UNIV. OF CALIFORNIA, BERKELEY \\ UNIV. OF CALIFORNIA, DAVIS \\ UNIV. OF CALIFORNIA, LOS ANGELES \\ UNIV. OF CALIFORNIA, RIVERSIDE \\ UNIV. OF CALIFORNIA, SAN DIEGO \\ UNIV. OF CALIF., SANTA BARBARA
}

\author{
Vyjayanthi Chari \\ Department of Mathematics \\ University of California \\ Riverside, CA 92521-0135 \\ chari@math.ucr.edu \\ Kefeng Liu \\ Department of Mathematics \\ University of California \\ Los Angeles, CA 90095-1555 \\ liu@math.ucla.edu \\ Jie Qing \\ Department of Mathematics \\ University of California \\ Santa Cruz, CA 95064 \\ qing@cats.ucsc.edu
}

These supporting institutions contribute to the cost of publication of this Journal, but they are not owners or publishers and have no responsibility for its contents or policies.

See inside back cover or msp.org/pjm for submission instructions.

The subscription price for 2013 is US \$400/year for the electronic version, and \$485/year for print and electronic.

Subscriptions, requests for back issues and changes of subscribers address should be sent to Pacific Journal of Mathematics, P.O. Box 4163, Berkeley, CA 94704-0163, U.S.A. The Pacific Journal of Mathematics is indexed by Mathematical Reviews, Zentralblatt MATH, PASCAL CNRS Index, Referativnyi Zhurnal, Current Mathematical Publications and the Science Citation Index.

The Pacific Journal of Mathematics (ISSN 0030-8730) at the University of California, c/o Department of Mathematics, 798 Evans Hall \#3840, Berkeley, CA 94720-3840, is published monthly except July and August. Periodical rate postage paid at Berkeley, CA 94704, and additional mailing offices. POSTMASTER: send address changes to Pacific Journal of Mathematics, P.O. Box 4163, Berkeley, CA 94704-0163.

PJM peer review and production are managed by EditFLOW ${ }^{\circledR}$ from Mathematical Sciences Publishers.

PUBLISHED BY

mathematical sciences publishers

nonprofit scientific publishing

http://msp.org/

(C) 2013 Mathematical Sciences Publishers 


\section{PACIFIC JOURNAL OF MATHEMATICS}

Volume $261 \quad$ No. $2 \quad$ February 2013

Geography of simply connected nonspin symplectic 4-manifolds with positive signature

Anar Akhmedov, Mark C. Hughes and B. Doug Park

Schur-Horn theorems in $\mathrm{II}_{\infty}$-factors

283

MARTÍN ARGERAMI and PEDRo MASSEY

Classification of positive solutions for an elliptic system with a higher-order

fractional Laplacian

JiNGBo DOU and CHANGZHENG QU

Bound states of asymptotically linear Schrödinger equations with compactly supported potentials

MingWEN FEI and HUICHENG YiN

Type I almost homogeneous manifolds of cohomogeneity one, III

DANIEL GUAN

The subrepresentation theorem for automorphic representations

MARCELA HANZER

Variational characterizations of the total scalar curvature and eigenvalues of the

Laplacian

Seungsu Hwang, JeOngwook Chang and GabJin Yun

Fill-ins of nonnegative scalar curvature, static metrics, and quasi-local mass

JEFFREY L. JAUREGUI

Operator algebras and conjugacy problem for the pseudo-Anosov automorphisms of a surface

\section{IGOR NIKOLAEV}

Connected sums of closed Riemannian manifolds and fourth-order conformal invariants

DAVID RASKE

Ruled minimal surfaces in the three-dimensional Heisenberg group

Heayong Shin, Young Wook Kim, Sung-Eun Koh, Hyung Yong LeE and SEONG-DEOG YANG

$G$-bundles over elliptic curves for non-simply laced Lie groups and configurations of lines in rational surfaces

MANG XU and JIAJIN ZHANG 